\title{
Facile And Direct Adhesive of Cellulose Substrates Using Inorganic Metal Salt Through Partial Welding
}

Heng Zhang ( $\nabla$ zhangheng0625@sina.com)

Kunming University of Science and Technology https://orcid.org/0000-0001-6377-0537

\section{Shumei Zhang}

Kunming University of Science and Technology

\section{Gaozhe Liu}

Kunming University of Science and Technology

\section{Xin Gao}

Kunming University of Science and Technology

\section{Xiaoning Tang}

Kunming University of Science and Technology

\section{Shaoyun Shan}

Kunming University of Science and Technology

\section{Lincai Peng}

Kunming University of Science and Technology

\section{Keli Chen}

Kunming University of Science and Technology

\section{Research Article}

Keywords: Cellulose welding, Cellulose adhesive, Partial dissolution, Zinc chloride

Posted Date: October 13th, 2021

DOI: https://doi.org/10.21203/rs.3.rs-935282/v1

License: (c) (1) This work is licensed under a Creative Commons Attribution 4.0 International License. Read Full License 


\section{Abstract}

In this study, we describe a method of cellulose substrates bonding through partial dissolution using low dosage of zinc chloride aqueous solvent as an adhesive. The results exhibited that the bonding area of strips was tough without any failure though the mechanical test, shrinks in solvent-diffusion area was the main reason that let to breakage. The study found that shrink phenomena in diffusion area can be avoided on a certain extent by the decrease in dosage of zinc chloride. Scanning electron microscopy and X-ray diffractometer revealed that the cellulose was partial dissolved and regenerated cellulose filled with the gaps between fibers, providing evidences of the macromolecular interlocking and entangling mechanism for combining strips. Additionally, X-ray photoelectron spectroscopy showed that a certain amount of zinc oxides was remained in the adhesive strips, demonstrating a difficulty of zinc ions to be completely removed due to their close combination with cellulose chains. This work provides an attractive attempt that partially dissolved cellulose was used as an adhesive, extending the application of cellulose welding and a new concept for bonding cellulose materials.

\section{Introduction}

Cellulose is the most abundant biopolymer in the world which has attracted global attention for its main properties of renewability and biodegradability. Due to the complex matrix which are constituted by interand intramolecular hydrogen bonds, cellulose is hardly dissolved in common solvents (Ajdary et al. 2020; Wang et al. 2020). Due to the rising of environmental and social concerns, cellulose-based products are widely used. On the other hand, adhesives plays a critical role in people's daily life such as gluing, repairing, and attaching (Liu et al. 2021). The materials usually form an adhesive bond by inducing the change in their mechanical properties through heat, polymerization of monomers, and evaporation of solvents (Cudjoe et al. 2018). Furthermore, the effect of bonding is mainly dependent on whether the connection is well established between the interfaces of adhesive and material.

Cellulosic substrate like paper can be easily bonded for their high surface energy porous solids (Ferreira et al. 2015). However, it was difficult to avoid the negative consequences of the use for cellulose adhesive, such as, interference with the recycling process, toxic elements, and biodegradation, was. Unfortunately, the application of many adhesives for cellulosic substrate, including melamine urea, phenol formaldehyde resin, starch, and wheat flour, have been limited by their disadvantages of genotoxic, potential carcinogenicity, and poor fungi-resistance (Chen et al. 2018). Furthermore, these cellulose adhesives also lead to a difficulty in cellulose recycling because the residual adhesive mixture will contaminate the final product ( $\mathrm{Li}$ et al. 2013; Ouadi et al. 2019). Therefore, it is quite necessary to explore an alternative adhesive with the characteristics of eco-friendly, non-biotoxicity, and organic solvent free.

Besides the hash mechanical treatment, dissolution is a relatively mild route to obtain the homogeneous and plastic cellulose materials (Hasani et al. 2013). Although there are many researchers targeted the development of novel cellulose solvents (Parthasarathi et al. 2015; El Seoud et al. 2020; Haron et al. 
2021), the traditional solvents are still owned a great potential application. One class of solvents used for cellulose dissolution are inorganic molten salts which belong to the nonderivative reagent (Burger et al. 2020). Among these inorganic molten salts, zinc chloride hydrate is one of the most widely-used solvents for its recyclable, inexpensive and green features (Leipner et al. 2000; Fischer et al. 2003). Recently, some researchers point out that the $\mathrm{ZnCl}_{2} \cdot n \mathrm{H}_{2} \mathrm{O}$ system is a true ionic liquid (IL) when the molecular formula [ $\left.\mathrm{Zn}\left(\mathrm{OH}_{2}\right)_{6}\right]\left[\mathrm{ZnCl}_{4}\right]$ in the case of $n=3$ (Bodachivskyi et al. 2019a; Bodachivskyi et al. 2019b). It is indicated that the understanding of mechanism about $\mathrm{ZnCl}_{2}$ behavior in cellulose suspension from this conventional technology still need to be further developed. Generally, it is known that the concentration of $\mathrm{ZnCl}_{2}$ solvent used in cellulose dissolution is usually higher than $63 \%(\mathrm{w} / \mathrm{w})$ (Fischer et al. 2003;

Schestakow et al. 2016). Based on the authors' previous study, however, it was found that the initial concentration of zinc chloride solvent could be reduced to $45 \%$ (w/w) (Zhang et al. 2019; Tang et al. 2021). It is indicative that the consumption of chemicals can be reduced almost $28.6 \%$ during the process of cellulose dissolution. Apparently, the reduction in dosage of chemicals have notable benefits on the energy conservation and pollutant control in cellulose dissolution manufacture. Therefore, it is believed that $\mathrm{ZnCl}_{2} / \mathrm{H}_{2} \mathrm{O}$ system still has a great potential for preparing functional cellulose materials.

Following the successive conception of all-polymer composites, all cellulose composites (ACCs) have been introduced recently (Nishino et al. 2004). ACCs are obtained through a new fabrication route with solvent-assisted isolation. The principle of ACC preparation is described as that the easily dissolved substances like amorphous of cellulose was used as a glue to bonding layers among the micro/nanofibers during the cellulose regeneration process (Yousefi et al. 2015). The bonding relies on the increase of joints between elementary fibrils through the mechanisms about cohesion and entanglements of molecular chains which is also called as cellulose welding. ${ }^{23}$ Hence, cellulose is utilized as both matrix and reinforcement in ACC matrix. Since the whole ACC materials was subject to chemical treatment uniformly, its morphological change can be easily controlled (Nishino et al. 2004; Nishino and Arimoto 2007; Zhang et al. 2016).

In this study, we employed a facile and mild method using the inorganic molten salt as cellulose solvent to obtain the bonding between cellulosic substrates through partial dissolution. To discuss the formation mechanism of cellulose bonding and structural change of ACC materials, the morphologies, crystalline texture, thermal stability, and chemical characterization were tested and analyzed. This study discovered that the bonding area of strips was strong enough that no breakage was found in all the mechanical test. Unlike the initially expecting, however, results exhibited the deformation of cellulose substrate caused by chemicals infiltration was the main reason that led to the reduction of mechanical strength.

\section{Experimental}

\section{Materials}


Bleached pine (Pinus kesiya) pulps were supplied by the Yunnan Yun-Jing Forestry \& Pulp Mill Co. Ltd. (Pu'er, Yunnan Province, China). Zinc chloride (GR, 99\%) was obtained from Aladdin Corporation (Shanghai, China), and heated at $105^{\circ} \mathrm{C}$ over 8 hours to remove the residual water prior to use. Sodium chlorite (CP, 80\%), glacial acetic acid (AR, 99.5\%), potassium hydroxide (AR, 90\%), and glycerol (AR, 99\%), were supplied by Aladdin Corporation and used without any further purification. The deionized (DI) water was utilized throughout this study.

\section{Methods}

\section{Cellulose preparation}

The pulp of bleached pine was suspended in DI water $(3 \%, \mathrm{w} / \mathrm{w})$, then the chemicals including sodium chlorite and glacial acetic acid were added at the ratio of 2.5:1 (w/v) for further delignification, the whole system was heated to $70 \pm 0.5^{\circ} \mathrm{C}$ for 60 min using water bath. The procedure was repeated for 3 times. The delignified slurry was washed with DI water until the filtrate $\mathrm{pH}$ was at about $7 \sim 8$. The delignified sample was air dried for $48 \mathrm{~h}$, then collected. After that, $20 \mathrm{~g}$ of holocellulose was suspended in $500 \mathrm{~mL}$ $10 \%$ potassium hydroxide solution and reacted for $12 \mathrm{~h}$ at room temperature. The final mixture of pure cellulose was filtrated, washed, air-dried, and then collected. The cellulose sheets were prepared by a standard sheet forming machine (Kumgai Riki Kogyo Co., Ltd., Japan), controlled as $60 \mathrm{~g} / \mathrm{m}^{2}$, air dried under room temperature overnight.

\section{Cellulose materials fusion}

The substance of cellulose sheets from pine pulp were cut to the dimensions of $10 \times 150 \mathrm{~mm} .1 \mathrm{~mL}$ of $\mathrm{ZnCl}_{2}$ water solvent $(20 \%, 30 \%, 40 \%$; w/w) was dripped on the superimposed part of two cellulose strips, as shown in Fig. 1. The dimensions of the superimposed part were $10 \times 10 \mathrm{~mm}$. The strips were transferred into an oven $\left(85^{\circ} \mathrm{C}\right)$ after the solvent was fully infiltrated the superimposed part. These cellulose strips were removed and hot pressed $\left(85^{\circ} \mathrm{C}, 0.02 \mathrm{Mpa}\right)$ when the surface of them became translucent and gel-like. After the partial dissolution process, the welded strips were carefully dripped with the DI water for $15 \mathrm{~min}$ for rinsing the $\mathrm{ZnCl}_{2}$. After the purification, the achieved strips were dried at $85{ }^{\circ} \mathrm{C}$ under $0.06 \mathrm{MPa}$ pressing using a standard paper sheet machine.

\section{Material characterization}

\section{Surface morphology}

Scanning electron microscopy (SEM) observation of the cellulose sheets were performed using a Nova Nanosem 450 (FEl, USA) at an accelerating voltage of $5.0 \mathrm{kV}$. The samples were placed onto a carbon disk that was attached to the stage, and observation areas were chosen randomly.

\section{Crystallinity}


Overall crystalline phases of samples were determined by XRD measurement on an X'Pert 3 powder diffractometer (PANalytical Co. Ltd. Netherlands). The samples were positioned on a glass sample holder. Radial scans of intensity were recorded at ambient condition over scattering $2 \theta$ angles from $3^{\circ}$ to $50^{\circ}$ (scanning rate $=13.77 \mathrm{~s} / \mathrm{step}$, step size $\left.=0.01313^{\circ}\right)$ using a Cu Ka radiation $(\lambda=1.5406 \mathrm{~nm})$, the operating voltage and filament current was $40 \mathrm{kV}$ and $40 \mathrm{~mA}$. The crystallinity index (Crl) was calculated based on the peak deconvolution results:

$$
\operatorname{CrI}(\%)=\frac{I_{200}-I_{\mathrm{am}}}{I_{200}} \times 100
$$

where $I_{200}$ represents the height of scattered intensity at the main peak, and $I_{a m}$ stands for the height of the minimum peak intensity. The total intensity of the (200) peak for cellulose I is taken near $22.5^{\circ} ; I_{a m}$ is the amorphous intensity taken near $18^{\circ}$ for cellulose I. All the $\mathrm{Crl}$ values in this study are calculated from the original data without subtracting background. XRD spectra were analyzed by means of Jade 6.0 (Haron et al. 2021; Xu et al. 2021).

\section{Thermal properties}

A Netzsch STA 449 F3 (Netzsch Scientific Instruments Trading Ltd, Germany) was used to perform the thermal stability. Thermograms of cellulose samples were recorded between $50^{\circ} \mathrm{C}$ and $800^{\circ} \mathrm{C}$ with a heating rate of $10^{\circ} \mathrm{C} / \mathrm{min}$ in an inert atmosphere maintained by a nitrogen flow of $50 \mathrm{~mL} / \mathrm{min}$. Proteus (Version 5.0.0) software was used for calculating the first derivatives of the thermograms (DTG), the percentage weight loss, and the decomposition temperatures.

\section{X-ray Photoelectron Spectroscopy analysis}

XPS investigation was performed to obtain detailed information of atomic percentages and chemical composition. A Thermo escalab 25Xi equipment (Thermo Fisher, USA) was used with a mono AI Ka X-ray source $(1486.6 \mathrm{eV})$. All the samples were tested under the room temperature.

\section{Mechanical properties}

Mechanical properties of cellulose strips were measured by a tensile testing machine, model 103A (Shenzhen Wance Testing Macnine Co. Ltd, China). The sample was tested in accordance with the ASTM standard method D882-97. The testing machine was fitted with a $1000 \mathrm{~N}$ load cell with a crosshead speed of $10 \mathrm{~mm} / \mathrm{min}$, and the initial distance between the grips was $50 \mathrm{mms}$, and measurements were performed under room temperature. Each experiment for testing the tensile strength $(\sigma)$, Young's modulus $\left(E^{\prime}\right)$, and elongation at break $(\varepsilon)$ was performed in five times, and the average values were reported.

\section{Results And Discussion}

Stress-strain cures of cellulose substrates with and without welding treatment were shown in Fig. 2. The initial cellulose strips from pine displayed a strength of $22.5 \mathrm{MPa}$, however, the values of its welded strips were showed to be a certain decrease from $0.1 \mathrm{MPa}$ (welded by $10 \% \mathrm{ZnCl}_{2}$ solvent) to $16.4 \mathrm{MPa}$ (welded 
by $40 \% \mathrm{ZnCl}_{2}$ solvent). In the meantime, the elongation values of the welded strips were $7.7 \%\left(10 \% \mathrm{ZnCl}_{2}\right.$ solvent), $8.3 \%$ (20\% $\mathrm{ZnCl}_{2}$ solvent), $4.4 \%$ (30\% $\mathrm{ZnCl}_{2}$ solvent), and $2.2 \%$ ( $10 \% \mathrm{ZnCl}_{2}$ solvent), respectively, which were lower than that of the initial one (7.3\%).

Through the observation, it was found that the bonding area was strong enough that no breakage was found. As a matter of fact, the real reason that was resulted in the reduction of tensile strength was the shrinks of external dimensions which was near by the welding area (signed as infiltration area), as shown in Fig. 2. This experimental phenomenon was consistent with that in the study of Ferreira's (Ferreira et al. 2015). It is well known that the exchange of solvent with non-solvent leads to a desolvation of the cellulose molecules, and to the reformation of their intra- and inter-molecular hydrogen bonds (Medronho and Lindman 2015). Furthermore, these exchanges of molecules in usually let to dimensional shrinks of cellulose gel during the regeneration, causing the structure unevenness of whole materials, as shown in Fig. 3 of welding process. Therefore, due to this change of the external structure, the stress force was concentrated in these shrinks, resulting in the fracture of strips. The uniformity of whole materials were hardly to maintain after the regional welding, thus, many studies were focused on the whole substrate treatment through surface selective dissolution or dissolved cellulose and micro-/nano-cellulose composition (Fujisawa et al.; Huber et al. 2012; Isobe et al. 2012; Yousefi et al. 2015; Zhang et al. 2016; Khakalo et al. 2019).

It was clear that the infiltration of chemicals affected the uniformity of cellulose substrate near its welding area, and brought about the reduction of mechanical property. Due to the excellent hydrophilicity of cellulose, it is scarcely possible to eliminate the permeable diffusion of aqueous solvent among cellulosic matrix (Bai et al. 2017). It seems that the unnecessary infiltration was weaken as the usage of chemicals reduction. Conventionally, $\mathrm{ZnCl}_{2}$ solvent needs relatively high concentration (at least $65 \%$, $\mathrm{w} / \mathrm{w}$ ) to form $\mathrm{ZnCl}_{2} \cdot n \mathrm{H}_{2} \mathrm{O}$ system in order to dissolve cellulose (Fischer and Thümmler 2010). Based on our previous study, however, the initial concentration of $\mathrm{ZnCl}_{2}$ can be reduced to $45 \%$, in this case, whether the dissolution of cellulose mainly depended upon the amount of water evaporation (Zhang et al. 2019). Hence, it is possible to reduce the metal -salt dosage in the welding process under the same mechanism.

In the current study, high concentration (or dosage) of $\mathrm{ZnCl}_{2}$ solvent brings a remarkable wrinkle on the experimental substrates, resulting in dimensional changes (the translucent parts from $C$ and $D$ in Fig. 2b) nearby the superimposed parts. Because this chemical was infiltrated out of the regional welding region with water, making their cellulose swollen and dissolved. Theoretically, the shrink of cellulose substrate was also occurred in the welding region, however, due to the compaction of multilayer substrate made this effect inapparent. Therefore, it was important to regulate the dosage of chemical solvent to avoid the un-necessary swelling or dissolving.

From Fig. 2, it was observed that the tensile strength of cellulose strips (treated by $10 \% \mathrm{ZnCl}_{2}$ ) obtained a relatively close value $(22.4 \mathrm{MPa})$ to that of the pristine one $(22.5 \mathrm{MPa})$. Hence, the mechanical strength of strip was barely affected during the wielding, implying that the structure of an un-welding part of the 
cellulose strip was relatively intact. This result was confirmed that low dosage chemicals have more advantages on cellulose-strip connection compared with the high dosage. Therefore, the subsequent stage of this study was focused on the effect of adhesive using $10 \% \mathrm{ZnCl}_{2}$ solvent $(\mathrm{w} / \mathrm{w})$.

To understand the bonding effect of cellulose strips via $\mathrm{ZnCl}_{2}$ aqueous solution, detailed microstructures of the welding, diffusion, and pristine areas were investigated by SEM images in Fig. 4. It was observed from Fig. 4 that the surface of welding area was compact, and gaps between fibers was filled with regenerated cellulose. The part of regenerated cellulose was acted as a glue that connected the strips closely like one integral matrix, as shown in Fig. 4 of the cross-section view. It was demonstrated that the new chain-chain association was established between the strip's surface after regeneration. It is well known that the dissolution was conducive to establish the new hydrogen bonding, however, the Coulomb forces, van der Waals interactions, and hydrophobic interactions of cellulose were also promoted after the dissolution (Medronho et al. 2012). Hence, the mechanical strength of a bonding area between strips was stronger than that of a single strip.

Compared with the images of welding area, it was clear that the connections by the regenerated cellulose as fillers between fibers was remarkably reduced in the diffused area. Specifically, wrinkles were observed on the surface of fibers, indicating that the regional fibers in this area have been partial dissolved or swollen. Due to the low usage of chemicals, however, zinc chloride/water system did not affect severely the main structure of fibers in diffusion area. Hence, it was indicated that small amount of inorganic metal salt barely damages cellulose fibers, only wrinkling in the surface of fibers. Meanwhile, it was obvious that the morphology of fibers in the rest part of strip were not affected by $\mathrm{ZnCl}_{2}$. The images of Fig. 4 exhibited that the fibers in pristine area were smooth, stiff, and loose; The boundaries among fibers were clear, implying that their connections were the same as the conventional paper, which were mainly dependent on hydrogen bonds and Van der Waals force (Hirn and Schennach 2015).

In order to insight into the crystalline change of the cellulose sheets before and after $\mathrm{ZnCl}_{2}$ adhesion, XRD was used for observation. Figure 5 shows the XRD patterns of the cellulose substrates from different areas of the same strip which was bonded by $10 \% \mathrm{ZnCl}_{2}$ solvent. The diffraction curve of cellulose from pristine area was exhibited to be typical characteristic of cellulose l, with the main peaks at approximately $22.5^{\circ}$ and broad peaks near $16.7^{\circ}$ and $14.9^{\circ}$, respectively (Oudiani et al. 2011; French 2014; Garemark et al. 2020). Differently, the diffraction curve of cellulose from welded area was showed to be classical characteristic of cellulose II with the main peaks at approximately $21.0^{\circ}$ and $12.0^{\circ}$, respectively (Chen et al. 2020; Zhang et al. 2018). The diffraction cure of cellulose from the diffusion area was more complicated compared with the others, since its pattern was exhibited to be a transition state of crystalline form from I to II (Tang et al. 2021).

Due to the intrusion of zinc ions into crystalline regions and destruction of the hydrogen bonds of the pristine network, it is no doubt that the crystalline type of cellulose was transformed during cellulose dissolution. Cellulose welding treatment, however, was belong to a class of partial dissolution, that is, the reaction was mainly happened in the $P$ wall and parts of S1 regions of fibers (Tang et al. 2021). 
Therefore, although conspicuous fiber structures were still observed in the paper sheet from Fig. 4, the XRD outcome of the welding area was a typical characteristic of cellulose II. Due to the free diffusion of zinc ions with water molecules in the cellulose substrate, partly zinc ions were moved into the diffusion area, and concentrated during the heating process. Hence, partial dissolution was also happened in this area, however, only surface of few fibers was affected in that the amount of zinc ions was comparatively low. As a matter of fact, most of these fibers in the diffusion region were just swelled. Thus, the crystalline form in the diffusion area exhibited a state of polycrystalline mixture, including both cellulose I and II. More specifically, the XRD pattern of the diffused cellulose exhibited an intensity reduction of the main peaks of cellulose I, meanwhile, an emergence of characteristic peak of cellulose II. Therefore, it was observed that the crystallinity index was decreased gradually in the adhesive strips from $77.44 \%$ for the pristine cellulose to $73.10 \%$ for the diffused cellulose and $68.82 \%$ for the welded cellulose, as displayed in the inserted table of Fig. 5.

The thermal degradation behavior of cellulose from the welded, diffused, and pristine areas in the bonded substrate was observed using TGA and DTG analysis, as shown in Fig. 6. Generally, the initial weight loss before $150{ }^{\circ} \mathrm{C}$ was due to the moisture evaporation, while the sharp drop of weight in the approximately rang at $250-380^{\circ} \mathrm{C}$ was associated with decomposition of crystal water and thermal degradation of cellulose backbones (Jia et al. 2017; D’Acierno et al. 2020).

Table 1

Thermal properties of a cellulose strip from the welded, diffused, and pristine areas.

\begin{tabular}{|c|c|c|c|c|c|c|c|}
\hline \multirow[t]{2}{*}{$\begin{array}{l}\text { Sample } \\
\text { from }\end{array}$} & \multicolumn{3}{|c|}{$\begin{array}{l}\text { TGA: degradation temp } \\
\left({ }^{\circ} \mathrm{C}\right)\end{array}$} & \multirow{2}{*}{$\begin{array}{l}\text { Char yield at } 600 \\
{ }^{\circ} \mathrm{C} \\
(\%)\end{array}$} & \multicolumn{3}{|c|}{$\begin{array}{l}\text { DTG: maximum temperature peaks } \\
\left({ }^{\circ} \mathrm{C}\right)\end{array}$} \\
\hline & $T_{10 \%}$ & $T_{50 \%}$ & $T_{\text {onset }}$ & & $25-150$ & $150-200$ & $200-400$ \\
\hline $\begin{array}{l}\text { Pristine } \\
\text { area }\end{array}$ & 286.3 & 341.0 & 275.4 & 26.5 & 72.5 & 192.8 & 333.7 \\
\hline $\begin{array}{l}\text { Diffused } \\
\text { area }\end{array}$ & 277.4 & 350.9 & 259.4 & 31.9 & 69.8 & 193.6 & 305.8 \\
\hline $\begin{array}{l}\text { Welded } \\
\text { area }\end{array}$ & 246.3 & 391.5 & 230.9 & 37.9 & nd & 193.4 & 271.0 \\
\hline \multicolumn{8}{|c|}{$\begin{array}{l}T_{10 \%}: 10 \% \text { weight loss temperature. } T_{50 \%}: 50 \% \text { weight loss temperature. } T_{\text {onset }}: \text { the temperature of } \\
\text { degradation occurs. nd: not dected. }\end{array}$} \\
\hline
\end{tabular}

Although the cellulose samples were released adsorbed water at low temperature $\left(<100{ }^{\circ} \mathrm{C}\right)$, they might still contain a slight amount of water, which could be noticed from the DTG curve, and the results was shown to be $150-200{ }^{\circ} \mathrm{C}$ in Table 1 . The reason of this phenomenon was due to the strong hydrophilicity of pine cellulose, in the meantime, the dense surface of pine fiber also obstructed the adsorbed water releasing in a certain extent. Thus, when the samples were heated up over $193{ }^{\circ} \mathrm{C}$, the absolutely dry state of the samples could be obtained ( $\mathrm{Li}$ et al. 2019). Compared with other samples, the welded cellulose exhibited more thermolability in the low temperature range $\left(<100^{\circ} \mathrm{C}\right)$ because there was no prominent weight loss. It was implied that the welded cellulose contained less adsorbed water than the other 
specimens due to its compact and uniform structure. During the main stage of thermal decomposition, it is well known that the thermal stability of cellulose I is much higher compared with cellulose II due to the changing of crystal form (Xing et al. 2018). According to the above XRD analysis (Figure 5), the pristine area with cellulose I resulted in its higher thermal stability than the welded area with crystal form of cellulose II. Furthermore, the cellulose from the diffused area was a mixed state containing both cellulose I and cellulose II, thus, its thermal stability was between the pristine and welded areas. Therefore, it was observed that the initial decomposition temperature $\left(T_{\text {onset }}\right)$ of samples was $230.9{ }^{\circ} \mathrm{C}$ (welded), $259.4{ }^{\circ} \mathrm{C}$ (diffused), and $275.4{ }^{\circ} \mathrm{C}$ (pristine), respectively.

The char weight at $800{ }^{\circ} \mathrm{C}$ of welded strips, interestingly, was $5.4-11.4 \%$ higher compared with the other samples, as exhibited in Table 1. While, the residual weight of the pristine sample was the lowest $(26.5 \%)$ among these samples. Combined with the infiltration of zinc ions in the welding process, it was believed that the change of char weight mainly depended on zinc ions remained in the sheets.

The evidence of zinc ions was appeared in cellulose welding strips as residue was exhibited in the TGA curves (Fig. 6). Thus, it was necessary to identify further the presence of zinc ions in the adhesive cellulose strips, XPS spectra were recorded, as shown in Fig. 7a. The XPS spectrum of welded and diffused area exhibited C 1s, 01 s, and Zn 2p peaks, indicating that the cellulose-Zn composite was obtained (Ma et al. 2016a; Li et al. 2020). Especially, the Zn 2p spectrum showed two peaks ( $Z n 2_{3 / 2}$ and $\mathrm{Zn} \mathrm{2} \mathrm{p}_{1 / 2}$ lines) with binding energies of 1023.3 and $1046.4 \mathrm{eV}$ in Fig. 7b (Ma et al. 2016b). The difference of binding energy was $23.4 \mathrm{eV}$ which was closely to the standard value of $\mathrm{ZnO}(23.0 \mathrm{eV})$ (Goktas and Goktas 2021). The result indicated that the zinc ions were mainly remained in the cellulose substrate as $\mathrm{ZnO}$. Base on the references, the $\mathrm{ZnO}$ can be obtained from $\mathrm{ZnCl}_{2}$ through hydrolysis reactions even at a relatively low temperature, describing by Eqs. (1) and (2) (Yang et al. 2011; Niazi et al. 2020).

$\mathrm{ZnCl}_{2}+2 \mathrm{H}_{2} \mathrm{O} \leftrightarrow \mathrm{Zn}(\mathrm{OH})_{2}+2 \mathrm{HCl}(1)$

$\mathrm{ZnCl}_{2}+\mathrm{H}_{2} \mathrm{O} \leftrightarrow \mathrm{ZnO}+2 \mathrm{HCl}(2)$

Due to the washing process, vast majority of $\mathrm{ZnCl}_{2}$ molecules were rinsed into the effluent, however, there were parts of zinc ions was formed as $\mathrm{ZnO}$ remained in the cellulose materials for their property of insoluble in the water. Based on the literatures, the binding energy at $532.9 \mathrm{eV}$ was characteristic of the 0 atom from alcoholic $\mathrm{C}-\mathrm{OH}$ groups in $\mathrm{ZnO}$-cellulose composite, whereas that at $532.2 \mathrm{eV}$ originated from the $\mathrm{O}$ atom of $\mathrm{ZnO}$ (Li et al. 2021; Kotsis and Staemmler 2006). In our study, the two peaks at 532.9 and $532.2 \mathrm{eV}$ were combined as one peak at $532.7 \mathrm{eV}$, shown in Fig. 7c. Thus, it was reconfirmed that there was $\mathrm{ZnO}$ substance in the cellulose welding strips. Furthermore, it was verified that there was interaction between $\mathrm{ZnO}$ and cellulose base on the Ma's reaserch (Ma et al. 2016a). Therefore, due to the interaction of cellulose-ZnO and slightly solubility of $\mathrm{ZnO}$ in water, it was clearly to understand that the zinc ions was hardly to remove from cellulose bonding strips in washing process.

\section{Conclusions}


$\mathrm{ZnCl}_{2}$ aqueous solution is a conventional, efficient, economical, and nontoxic solvent used for cellulose dissolution. In this study, cellulose strips were bonding together through the partial dissolution using $\mathrm{ZnCl}_{2}$ solvent as an adhesive. Due to the new entanglement of cellulose chains shrinks were appeared in the substrate, leading to its dimension change. It was indicated that low usage of $\mathrm{ZnCl}_{2}$ was suitable for bonding cellulose strips, and considering the maintenance of the mechanical strength. Regenerated cellulose was filled and formed between undissolved fibers as a glue which contributed to the effect of adhesion as the van der Waals, electrostatic, and hydrogen bonds were enforced. The structure of the whole strip was integrity and barely shrink, even in the solvent diffusion area, and the concentration of stress force was hardly caused. It was expected that $\mathrm{ZnCl}_{2}$ welding cellulose could provide a novel and simple technology to well bond cellulose-based materials.

\section{Declarations}

\section{Acknowledgments}

This study was financially supported by the National Natural Science Foundation of China (51963012), Science Research Foundation Project of Yunnan Municipal Education Commission (2018js027), and KMUST Scientific Research Foundation for the Introduction of Talent (KKZ3202005045).

\section{References}

1. Ajdary R, Tardy BL, Mattos BD et al (2020) Plant Nanomaterials and Inspiration from Nature: Water Interactions and Hierarchically Structured Hydrogels. Adv Mater 2001085:. https://doi.org/10.1002/adma.202001085

2. Bai L, Bossa N, Qu F et al (2017) Comparison of Hydrophilicity and Mechanical Properties of Nanocomposite Membranes with Cellulose Nanocrystals and Carbon Nanotubes. Environ Sci Technol 51:253-262. https://doi.org/10.1021/acs.est.6b04280

3. Bodachivskyi I, Kuzhiumparambil U, Williams DBG (2019a) The role of the molecular formula of $\mathrm{ZnCl} 2 \because \mathrm{N} \mathrm{H} 2 \mathrm{O}$ on its catalyst activity: A systematic study of zinc chloride hydrates in the catalytic valorisation of cellulosic biomass. Catal Sci Technol 9:4693-4701. https://doi.org/10.1039/c9cy00846b

4. Bodachivskyi I, Kuzhiumparambil U, Williams DBG (2019b) Acid-Catalysed Conversion of Carbohydrates into Furan-Type Molecules in Zinc Chloride Hydrate. Chempluschem 84:352-357. https://doi.org/10.1002/cplu.201800650

5. Burger D, Winter A, Subbiahdoss G et al (2020) Partial Amorphization of Cellulose through Zinc Chloride Treatment: A Facile and Sustainable Pathway to Functional Cellulose Nanofibers with Flame-Retardant and Catalytic Properties. ACS Sustain Chem Eng 8:13576-13582. https://doi.org/10.1021/acssuschemeng.0c03492 
6. Chen T, Wu Z, Wang XA et al (2018) Hierarchical Lamellar Aluminophosphate Materials with Porosity as Ecofriendly Inorganic Adhesive for Wood-Based Boards. ACS Sustain Chem Eng 6:6273-6280. https://doi.org/10.1021/acssuschemeng.8b00078

7. Chen Y, Yu HY, Li Y (2020) Highly Efficient and Superfast Cellulose Dissolution by Green Chloride Salts and Its Dissolution Mechanism. ACS Sustain Chem Eng 8:18446-18454. https://doi.org/10.1021/acssuschemeng.0c05788

8. Cudjoe E, Herbert KM, Rowan SJ (2018) Strong, Rebondable, Dynamic Cross-Linked Cellulose Nanocrystal Polymer Nanocomposite Adhesives. ACS Appl Mater Interfaces 10:30723-30731. https://doi.org/10.1021/acsami.8b10520

9. D'Acierno F, Hamad WY, Michal CA, Maclachlan MJ (2020) Thermal Degradation of Cellulose Filaments and Nanocrystals. Biomacromol 21:3374-3386. https://doi.org/10.1021/acs.biomac.0c00805

10. El Seoud OA, Kostag M, Jedvert K, Malek NI (2020) Cellulose Regeneration and Chemical Recycling: Closing the "Cellulose Gap" Using Environmentally Benign Solvents. Macromol Mater Eng 305:1-21. https://doi.org/10.1002/mame.201900832

11. Ferreira ES, Lanzoni EM, Costa CAR et al (2015) Adhesive and Reinforcing Properties of Soluble Cellulose: A Repulpable Adhesive for Wet and Dry Cellulosic Substrates. ACS Appl Mater Interfaces 7:18750-18758. https://doi.org/10.1021/acsami.5b05310

12. Fischer $\mathrm{S}$, Leipner $\mathrm{H}$, Thümmler $\mathrm{K}$ et al (2003) Inorganic molten salts as solvents for cellulose. Cellulose 10:227-236. https://doi.org/10.1023/A:1025128028462

13. Fischer S, Thümmler K (2010) Molten inorganic salts as reaction medium for cellulose. ACS Symp Ser 1033:91-101. https://doi.org/10.1021/bk-2010-1033.ch004

14. French $A D$ (2014) Idealized powder diffraction patterns for cellulose polymorphs. Cellulose 21:885896. https://doi.org/10.1007/s10570-013-0030-4

15. Fujisawa S, Saito T, Isogai A, All-Cellulose (Cellulose - Cellulose) Green Composites

16. Garemark J, Yang X, Sheng X et al (2020) Top-Down Approach Making Anisotropic Cellulose Aerogels as Universal Substrates for Multifunctionalization. ACS Nano 14:7111-7120. https://doi.org/10.1021/acsnano.0c01888

17. Goktas S, Goktas A (2021) A comparative study on recent progress in efficient ZnO based nanocomposite and heterojunction photocatalysts: A review. J Alloys Compd 863:. https://doi.org/10.1016/j.jallcom.2021.158734

18. Haron GAS, Mahmood H, Noh MH et al (2021) Ionic Liquids as a Sustainable Platform for Nanocellulose Processing from Bioresources: Overview and Current Status. ACS Sustain Chem Eng 9:1008-1034. https://doi.org/10.1021/acssuschemeng.0c06409

19. Hasani M, Henniges U, Idström A et al (2013) Nano-cellulosic materials: The impact of water on their dissolution in DMAc/LiCl. Carbohydr Polym 98:1565-1572. https://doi.org/10.1016/j.carbpol.2013.07.001 
20. Hirn U, Schennach R (2015) Comprehensive analysis of individual pulp fiber bonds quantifies the mechanisms of fiber bonding in paper. Sci Rep 5:1-9. https://doi.org/10.1038/srep10503

21. Huber T, Müssig J, Curnow $O$ et al (2012) A critical review of all-cellulose composites. J Mater Sci 47:1171-1186. https://doi.org/10.1007/s10853-011-5774-3

22. Isobe N, Kimura S, Wada M, Kuga S (2012) Mechanism of cellulose gelation from aqueous alkaliurea solution. Carbohydr Polym 89:1298-1300. https://doi.org/10.1016/j.carbpol.2012.03.023

23. Jia C, Bian H, Gao T et al (2017) Thermally Stable Cellulose Nanocrystals toward High-Performance 2D and 3D Nanostructures. ACS Appl Mater Interfaces 9:28922-28929.

https://doi.org/10.1021/acsami.7b08760

24. Khakalo A, Tanaka A, Korpela A et al (2019) All-Wood Composite Material by Partial Fiber Surface Dissolution with an lonic Liquid. ACS Sustain Chem Eng 7:3195-3202. https://doi.org/10.1021/acssuschemeng.8b05059

25. Kotsis K, Staemmler V (2006) Ab initio calculations of the 01s XPS spectra of ZnO and Zn oxo compounds. Phys Chem Chem Phys 8:1490-1498. https://doi.org/10.1039/B515699H

26. Leipner H, Fischer S, Brendler E, Voigt W (2000) Structural changes of cellulose dissolved in molten salt hydrates. Macromol Chem Phys 201:2041-2049. https://doi.org/10.1002/15213935(20001001)201:15<2041::AID-MACP2041>3.0.CO;2-E

27. Li B, Hu J, Xiong H, Xiao Y (2020) Application and Properties of Microporous Carbons Activated by ZnCl2: Adsorption Behavior and Activation Mechanism. ACS Omega 5:9398-9407. https://doi.org/10.1021/acsomega.0c00461

28. Li B, Wang Z, Wu S et al (2013) Effects of adhesive aging on the characteristics of stickies and their removal during paper recycling. Ind Eng Chem Res 52:9698-9704.

https://doi.org/10.1021/ie401480u

29. Li W, Hu J, Cheng L et al (2019) Study on thermal behavior of regenerated micro-crystalline cellulose containing slight amount of water induced by hydrogen-bonds transformation. Polymer 185:121989. https://doi.org/10.1016/j.polymer.2019.121989

30. Li X, Li H, Wang X et al (2021) Facile in situ fabrication of ZnO-embedded cellulose nanocomposite films with antibacterial properties and enhanced mechanical strength via hydrogen bonding interactions. Int J Biol Macromol 183:760-771. https://doi.org/10.1016/j.ijbiomac.2021.04.175

31. Liu H, Feng Y, Cao X et al (2021) Chitin Nanocrystals as an Eco-friendly and Strong Anisotropic Adhesive. ACS Appl Mater Interfaces 13:11356-11368. https://doi.org/10.1021/acsami.1c02000

32. Ma J, Sun Z, Wang Z, Zhou X (2016a) Preparation of ZnO-cellulose nanocomposites by different cellulose solution systems with a colloid mill. Cellulose 23:3703-3715. https://doi.org/10.1007/s10570-016-1081-0

33. Ma J, Zhu W, Tian Y, Wang Z (2016b) Preparation of Zinc Oxide-Starch Nanocomposite and Its Application on Coating. Nanoscale Res Lett 11:. https://doi.org/10.1186/s11671-016-1404-y

34. Mashkour M, Tajvidi M, Kimura F et al (2014) Strong highly anisotropic magnetocellulose nanocomposite films made by chemical peeling and in situ welding at the interface using an ionic 
liquid. ACS Appl Mater Interfaces 6:8165-8172. https://doi.org/10.1021/am500709t

35. Medronho B, Lindman B (2015) Brief overview on cellulose dissolution/regeneration interactions and mechanisms. Adv Colloid Interface Sci 222:502-508. https://doi.org/10.1016/j.cis.2014.05.004

36. Medronho B, Romano A, Miguel MG et al (2012) Rationalizing cellulose (in)solubility: Reviewing basic physicochemical aspects and role of hydrophobic interactions. Cellulose 19:581-587. https://doi.org/10.1007/s10570-011-9644-6

37. Niazi S, Olsen E, Nygård HS (2020) Hydrolysis of eutectic compositions in the $\mathrm{ZnCl} 2: \mathrm{KCl}: \mathrm{NaCl}$ ternary system and effect of adding ZnO. J Mol Liq 317:114069. https://doi.org/10.1016/j.molliq.2020.114069

38. Nishino T, Arimoto N (2007) All-cellulose composite prepared by selective dissolving of fiber surface. Biomacromol 8:2712-2716. https://doi.org/10.1021/bm0703416

39. Nishino T, Matsuda I, Hirao K (2004) All-cellulose composite. Macromolecules 37:7683-7687. https://doi.org/10.1021/ma049300h

40. Ouadi M, Fivga A, Jahangiri $\mathrm{H}$ et al (2019) A Review of the Valorization of Paper Industry Wastes by Thermochemical Conversion. Ind Eng Chem Res 58:15914-15929. https://doi.org/10.1021/acs.iecr.9b00635

41. Oudiani A, El, Chaabouni Y, Msahli S, Sakli F (2011) Crystal transition from cellulose i to cellulose II in $\mathrm{NaOH}$ treated Agave americana L. fibre. Carbohydr Polym 86:1221-1229. https://doi.org/10.1016/j.carbpol.2011.06.037

42. Parthasarathi R, Balamurugan $\mathrm{K}$, Shi J et al (2015) Theoretical Insights into the Role of Water in the Dissolution of Cellulose Using IL/Water Mixed Solvent Systems. J Phys Chem B 119:14339-14349. https://doi.org/10.1021/acs.jpcb.5b02680

43. Schestakow M, Karadagli I, Ratke $L$ (2016) Cellulose aerogels prepared from an aqueous zinc chloride salt hydrate melt. Carbohydr Polym 137:642-649. https://doi.org/10.1016/j.carbpol.2015.10.097

44. Tang X, Liu G, Zhang H et al (2021) Facile preparation of all-cellulose composites from softwood, hardwood, and agricultural straw cellulose by a simple route of partial dissolution. Carbohydr Polym 256:. https://doi.org/10.1016/j.carbpol.2020.117591

45. Wang J, Zhang D, Chu F (2020) Wood-Derived Functional Polymeric Materials. Adv Mater 2001135:1-21. https://doi.org/10.1002/adma.202001135

46. Xing L, Gu J, Zhang W et al (2018) Cellulose I and II nanocrystals produced by sulfuric acid hydrolysis of Tetra pak cellulose I. Carbohydr Polym 192:184-192. https://doi.org/10.1016/j.carbpol.2018.03.042

47. Xu D, Wang S, Berglund LA, Zhou Q (2021) Surface Charges Control the Structure and Properties of Layered Nanocomposite of Cellulose Nanofibrils and Clay Platelets. ACS Appl Mater Interfaces 13:. https://doi.org/10.1021/acsami.0c18594

48. Yang R, Zheng J, Li W et al (2011) Low-temperature growth of ZnO nanostructures by oxygen plasma oxidation of $\mathrm{ZnCl} 2$. Mater Chem Phys 129:693-695. 
https://doi.org/10.1016/j.matchemphys.2011.05.027

49. Yousefi H, Mashkour M, Yousefi R (2015) Direct solvent nanowelding of cellulose fibers to make allcellulose nanocomposite. Cellulose 22:1189-1200. https://doi.org/10.1007/s10570-015-0579-1

50. Zhang H, Chen K, Gao X et al (2019) Improved thermal stability of regenerated cellulose films from corn (Zea mays)stalk pith using facile preparation with low-concentration zinc chloride dissolving. Carbohydr Polym 217:190-198. https://doi.org/10.1016/j.carbpol.2018.10.107

51. Zhang J, Zhang J, Luo N et al (2016) All-Cellulose Nanocomposites Reinforced with in Situ Retained Cellulose Nanocrystals during Selective Dissolution of Cellulose in an lonic Liquid. ACS Sustain Chem Eng 4:4417-4423. https://doi.org/10.1021/acssuschemeng.6b01034

52. Zhang XF, Hou T, Chen J et al (2018) Facilitated Transport of CO2 Through the Transparent and Flexible Cellulose Membrane Promoted by Fixed-Site Carrier. ACS Appl Mater Interfaces 10:2493024936. https://doi.org/10.1021/acsami.8b07309

\section{Figures}
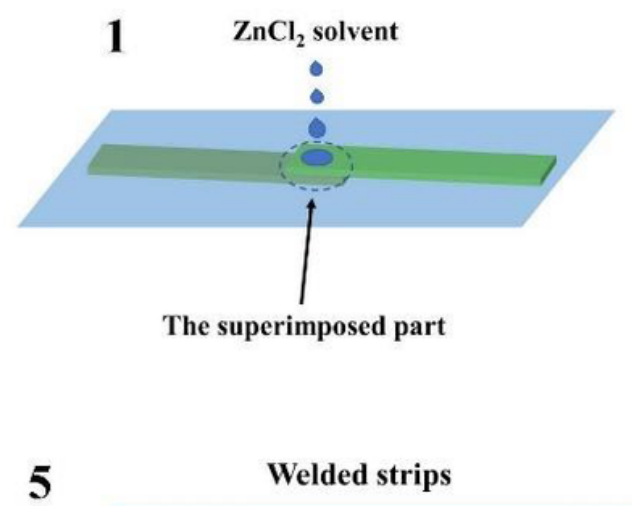

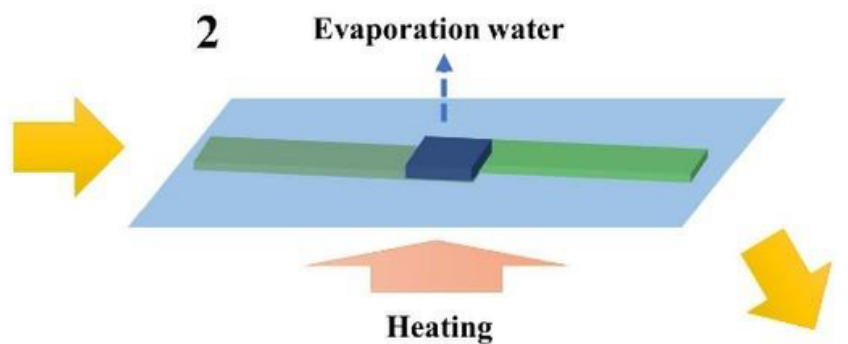

Hot pressing

\section{3}
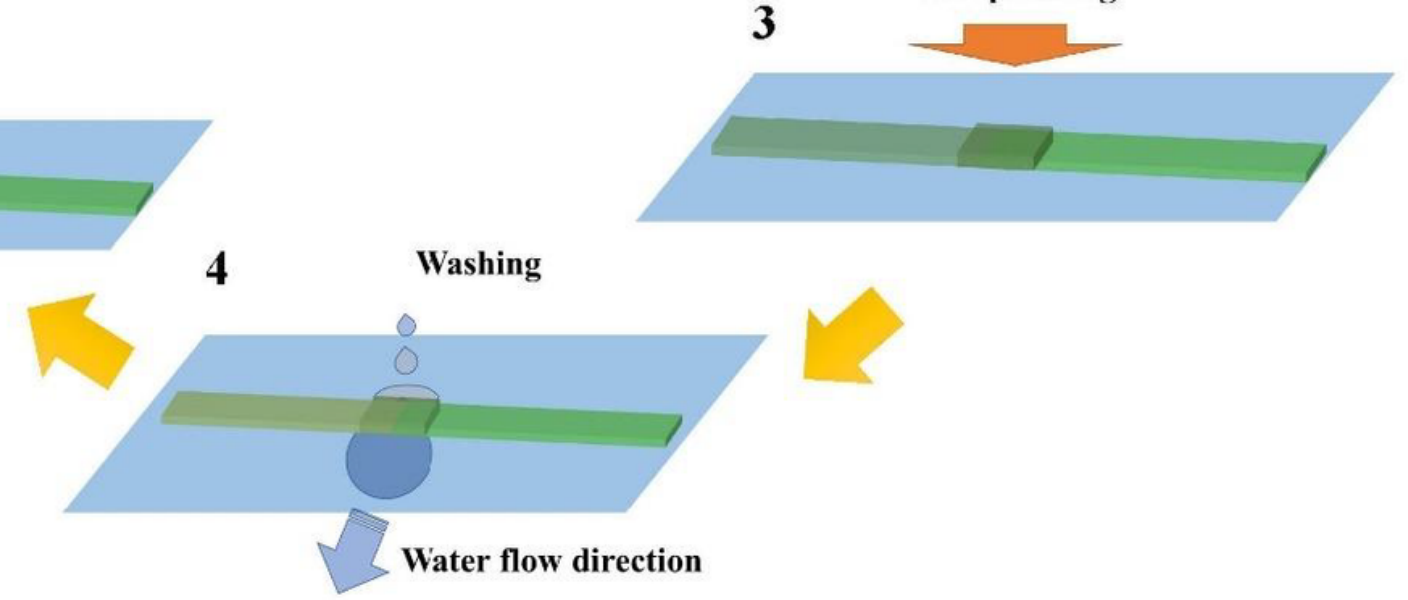

\section{Figure 1}

The schematic illustration of cellulose welding. 

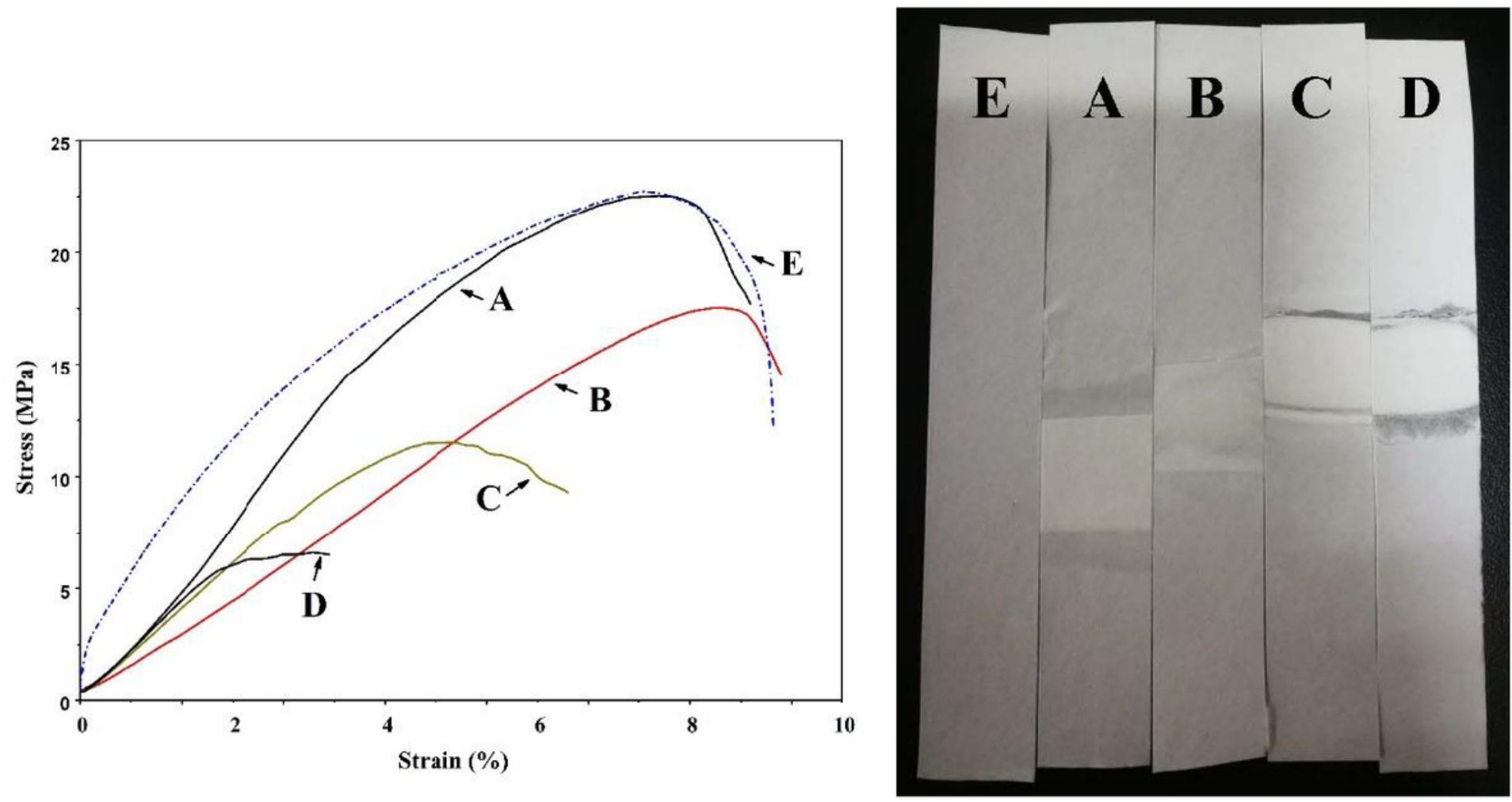

Figure 2

Stress-strain curves of the welded and initial cellulose strips, and their digital photos: (A) $10 \%$ (B) $20 \%$ (C) $30 \%$ (D) $40 \%$ and (E) initial.

\section{Infiltration process}

\section{Welding process}

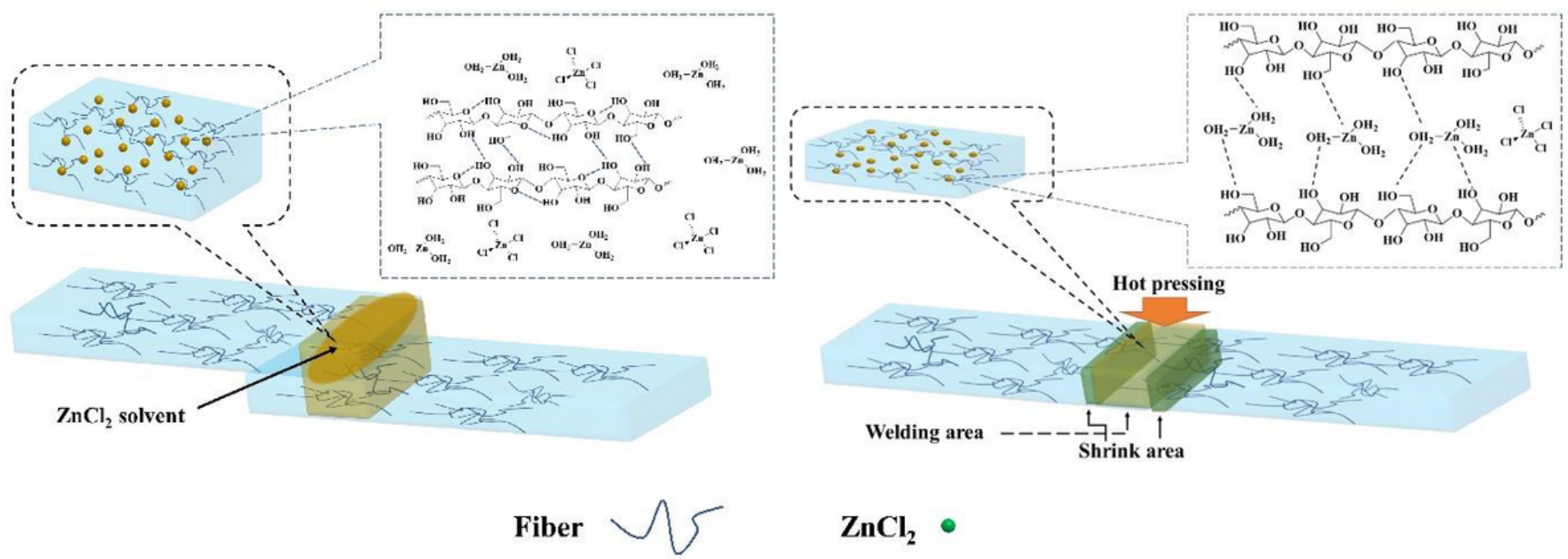

Figure 3

Schematic diagram of the welding part of cellulose strips. 


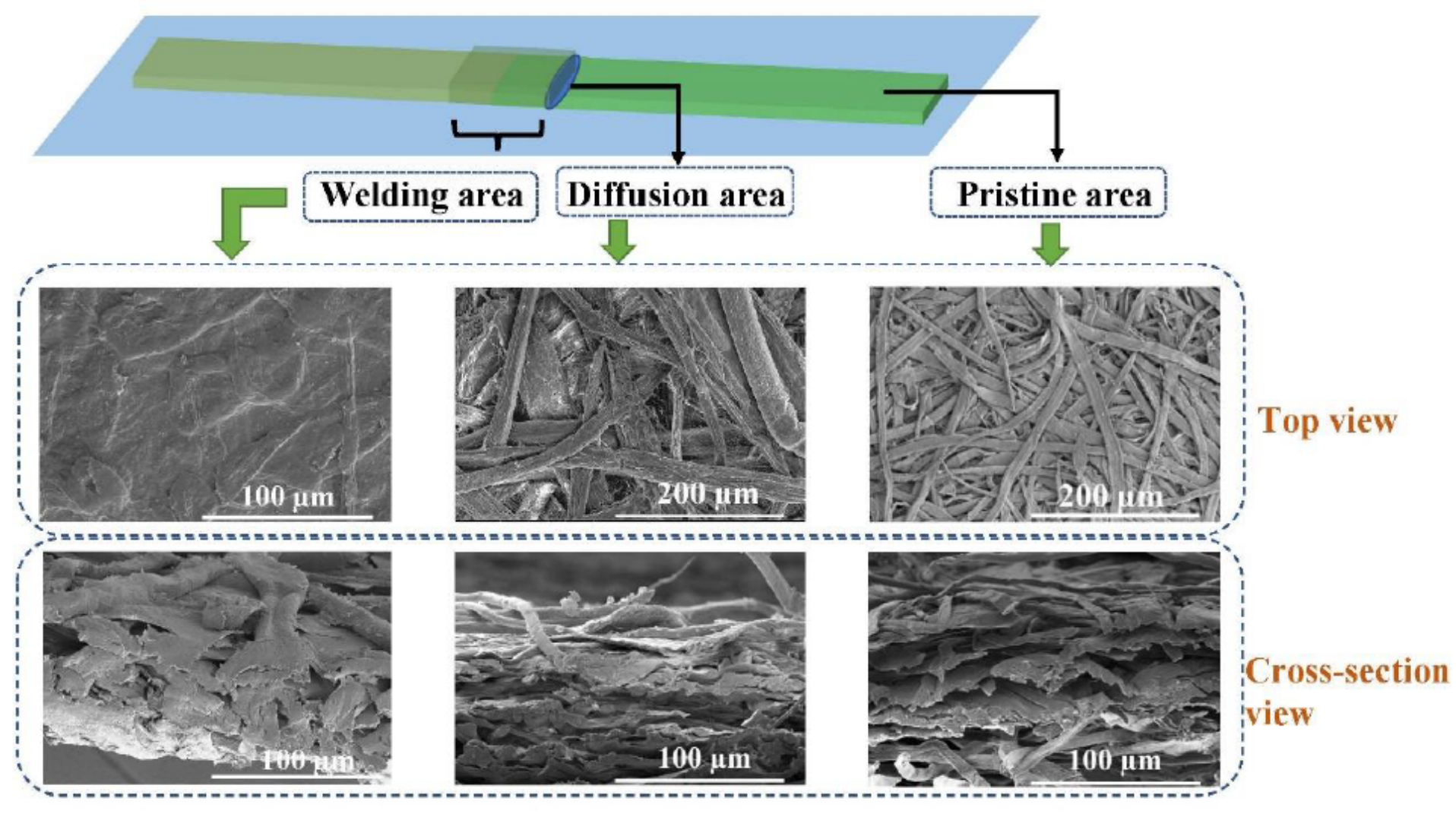

Figure 4

SEM observation of top and cross-section views from the pristine, welded, and diffused parts of the cellulose strips.

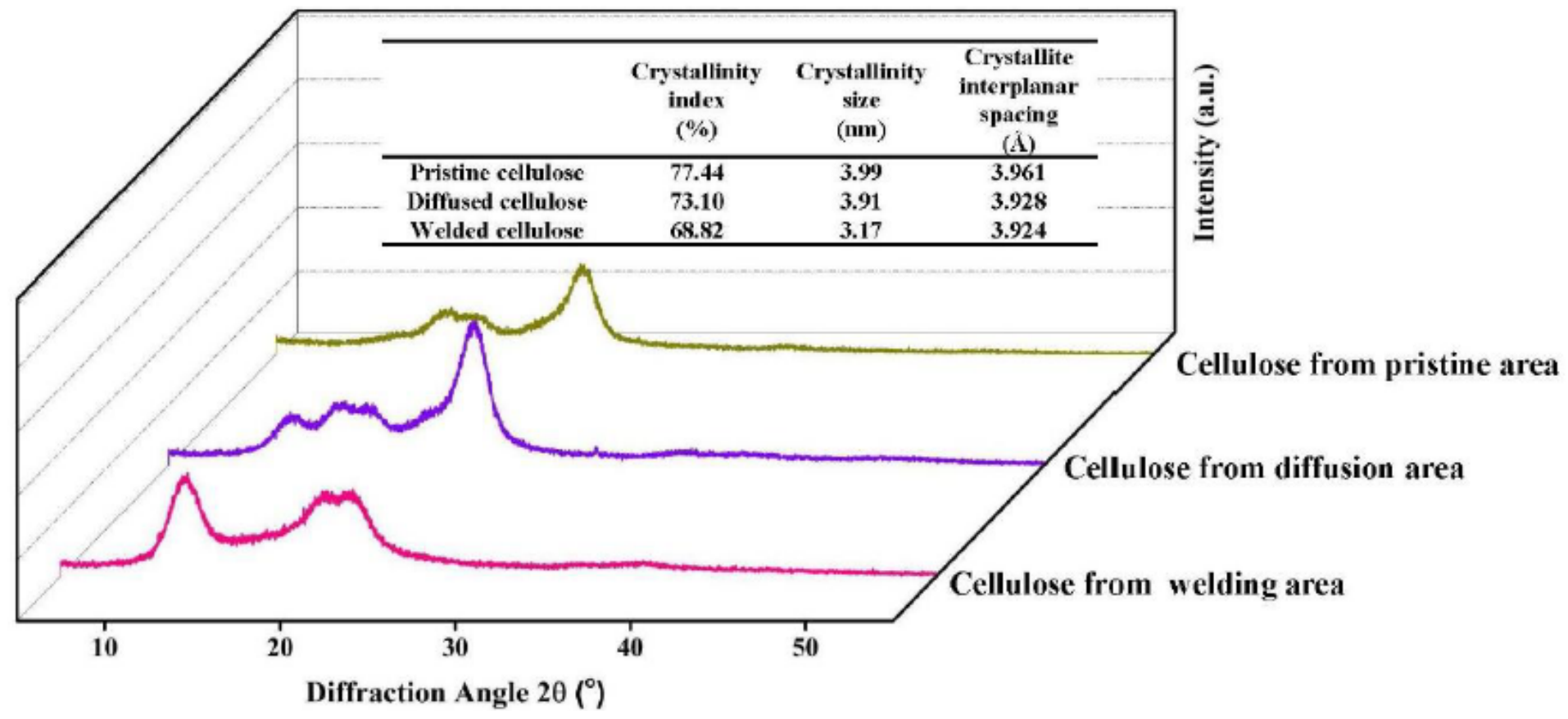

Figure 5 
XRD patterns of the cellulose substrates from welded, diffused, and pristine areas.
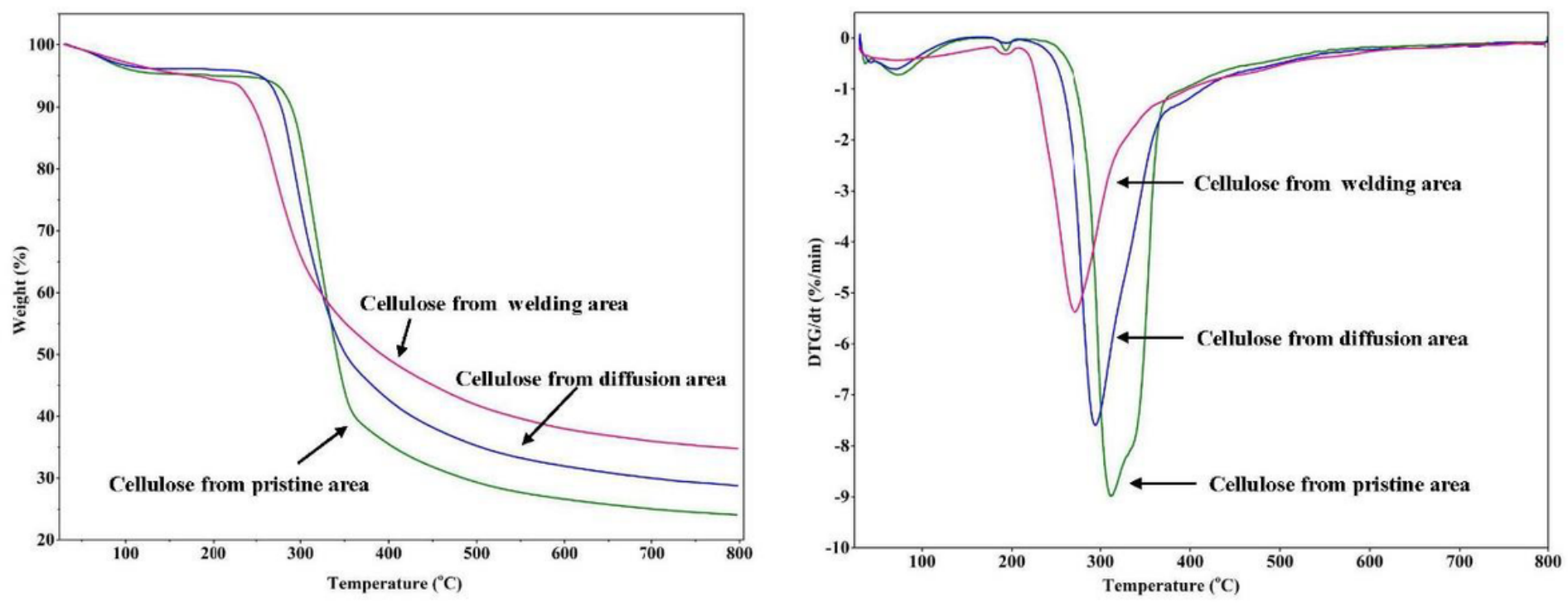

Figure 6

TGA and DTG curves of a cellulose strip from the welded, diffused, and pristine areas.
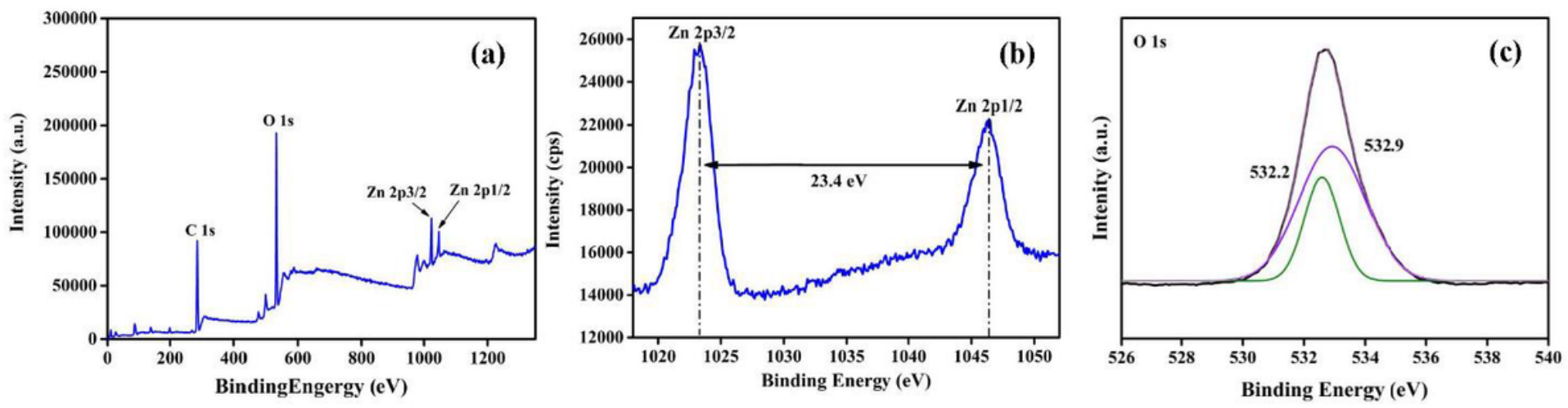

Figure 7

XPS spectra of the welding area from a cellulose strip: (a) wide-range spectra (b) Zn 2p region, and (c) 0 1 s region. 